\title{
A model to predict the risk of lethal nasopharyngeal necrosis after re-irradiation with intensity-modulated radiotherapy in nasopharyngeal carcinoma patients
}

\author{
Ya-Hui Yu ${ }^{1,2 \dagger}$, Wei-Xiong Xia ${ }^{1,2 \dagger}$, Jun-Li Shi ${ }^{1,3 \dagger}$, Wen-Juan Ma ${ }^{1,4}$, Yong Li ${ }^{1,5}$, Yan-Fang Ye ${ }^{6}$, Hu Liang ${ }^{1,2}$, \\ Liang-Ru Ke ${ }^{1,2}$, Xing L Lv ${ }^{1,2}$, Jing Yang ${ }^{1,2}$, Yan-Qun Xiang ${ }^{1,2^{*}}$ and Xiang Guo ${ }^{1,2^{*}}$
}

\begin{abstract}
Background: For patients with nasopharyngeal carcinoma (NPC) who undergo re-irradiation with intensity-modulated radiotherapy (IMRT), lethal nasopharyngeal necrosis (LNN) is a severe late adverse event. The purpose of this study was to identify risk factors for LNN and develop a model to predict LNN after radical re-irradiation with IMRT in patients with recurrent NPC.

Methods: Patients who underwent radical re-irradiation with IMRT for locally recurrent NPC between March 2001 and December 2011 and who had no evidence of distant metastasis were included in this study. Clinical characteristics, including recurrent carcinoma conditions and dosimetric features, were evaluated as candidate risk factors for LNN. Logistic regression analysis was used to identify independent risk factors and construct the predictive scoring model.

Results: Among 228 patients enrolled in this study, 204 were at risk of developing LNN based on risk analysis. Of the 204 patients treated, 31 (15.2\%) developed LNN. Logistic regression analysis showed that female sex $(P=0.008)$, necrosis before re-irradiation $(P=0.008)$, accumulated total prescription dose to the gross tumor volume $(\mathrm{GTV}) \geq 145.5 \mathrm{~Gy}(P=0.043)$, and recurrent tumor volume $\geq 25.38 \mathrm{~cm}^{3}(P=0.009)$ were independent risk factors for LNN. A model to predict LNN was then constructed that included these four independent risk factors.

Conclusions: A model that includes sex, necrosis before re-irradiation, accumulated total prescription dose to GTV, and recurrent tumor volume can effectively predict the risk of developing LNN in NPC patients who undergo radical re-irradiation with IMRT.
\end{abstract}

Keywords: Nasopharyngeal carcinoma, Re-irradiation, Intensity-modulated radiotherapy, Necrosis

\section{Background}

In South China, especially in Guangdong Province, nasopharyngeal carcinoma (NPC) is a common head and neck cancer $[1,2]$. It is a highly radiosensitive malignancy with a local recurrence rate of $10 \%-36 \%$ [3-5]. In

${ }^{*}$ Correspondence: xiangyq@sysucc.org.cn; guoxiang1010@gmail.com

${ }^{\dagger}$ Ya-Hui Yu, Wei-Xiong Xia and Jun-Li Shi contributed equally to this work

${ }^{2}$ Department of Nasopharyngeal Carcinoma, Sun Yat-sen University

Cancer Center, Guangzhou 510060, Guangdong, P. R. China

Full list of author information is available at the end of the article the past several decades, various treatment modalities, including external-beam radiotherapy (EBRT), intracavitary brachytherapy, interstitial radioactive implantation, stereotactic radiosurgery, nasopharyngectomy, chemotherapy, and a combination of these methods, have been used to treat the patients with NPC. Each method has its optimal treatment population [6]; for patients with locally recurrent NPC, EBRT remains the most effective modality [7].

Intensity-modulated radiotherapy (IMRT) is considered a better technique for the treatment of recurrent 
NPC because of its lower normal tissue doses and more homogeneous target doses compared with conformal radiotherapy [8]. Currently, radical IMRT is the most effective treatment for patients with locally recurrent NPC. However, IMRT does carry a risk of late toxicities that should not be ignored. For patients re-irradiated with IMRT for recurrent NPC, the patients with irreversible nasopharyngeal necrosis have the largest proportion of reported severe late adverse events [9], which may result in massive nasopharyngeal hemorrhage and death. Therefore, for patients with recurrent NPC who have undergone re-irradiation, it is urgent to predict and avoid lethal nasopharyngeal necrosis (LNN). In this retrospective study, we examined the relationship between the clinical characteristics of re-irradiated NPC patients and the incidence of LNN, and we subsequently developed a predictive scoring model for LNN.

\section{Patients and methods}

\section{Patient selection}

Patients who underwent radical re-irradiation with IMRT for locally recurrent NPC between March 2001 and December 2011 and who had no evidence of distant metastasis were included in this study. All patients had weekly nasopharyngeal examinations with nasopharyngoscopy during IMRT treatment and were followed 1 month after IMRT, every 3 months for the first 2 years, every 6 months for 3 additional years, and annually thereafter. Each follow-up included a complete medical record review, physical examination, and nasopharyngoscopy examination. At least once a year, if necessary, patients underwent serum electrolyte and complete blood count tests as well as chest X-ray, ultrasound/computed tomography $(\mathrm{CT})$ of the abdomen, and magnetic resonance imaging (MRI) of the head-and-neck region. This study was approved by the Ethics Committee of Sun Yat-sen University Cancer Center.

IMRT was conducted as previously reported [10, 11]. Patients were placed in a supine position on a custommade head support and immobilized with a thermoplastic mask covering the head and upper neck. CT scanning from the superior border of the frontal sinus to $2 \mathrm{~cm}$ below the clavicular heads was performed with a 3-mm slice thickness. Imaging data were transferred to the Corvus inverse treatment planning system (NOMOS Corporation, Wexford, PA, USA). The gross tumor volume (GTV), clinical target volume (CTV), gross tumor volume of cervical lymph node (GTVnd), and organs at risk (OARs) were contoured slice by slice on $\mathrm{CT}$ images. The recurrent gross target of the nasopharynx and lymph nodes in the neck were determined according to CT/ MRI and physical examinations. According to guidelines from the International Commission on Radiation Units and Measurements Report 62 (ICRU62) [12], CTV was defined as GTV plus a $0.5-1 \mathrm{~cm}$ margin; it also included the recurrent lymph nodes in the neck. OARs, including the brainstem, spinal cord, temporal lobes, lens, optic nerves, chiasm, parotid glands, temporomandibular joints, and mandible, were carefully contoured. Planning target volumes for GTV, GTVnd, and CTV were generated according to immobilization and localization uncertainties. According to our measurements, the immobilization uncertainty was $2 \mathrm{~mm}$ in the lateral direction and $1 \mathrm{~mm}$ in the anterior, posterior, superior, and inferior directions. Localization uncertainty was defined according to the involved organs' mobility, which was 1-2 $\mathrm{mm}$ for both tumor targets and the surrounding normal tissues. The total uncertainty, as automatically generated by the Corvus system, was $1.4-2.8 \mathrm{~mm}$ in every orientation. All patients received full-course IMRT with 6-MV X-rays generated by a Clinac-600C linear accelerator (Varian Medical Systems, Palo Alto, CA, USA). As a general rule, the dose comprising 33\% of contralateral OARs was limited to less than one-half of the tolerance dose, referring to a severe complication rate of $5 \%$ within 5 years of radiotherapy (TD5/5). The dose of the ipsilateral side was not constrained as that of the contralateral side. Dose verification was carried out before re-irradiation. The dose error between the measurement and the plan was less than $3 \%$.

\section{Diagnosis of nasopharyngeal necrosis}

Diagnosis of nasopharyngeal necrosis was based on the clinical characteristics, including foul nasal smell, refractory headache, filemot necrotic tissue and skull base osteoradionecrosis in nasopharyngeal cavity under endoscopy, discontinuous nasopharyngeal mucosa line and/or tissue defects on MRI, and a heap of red-stained substance without cellular structure in hematoxylineosin staining under pathologic examination [11, 13-19]. Patients who died from intractable epistaxis diagnosed with nasopharyngeal necrosis were recorded as having LNN.

\section{Study parameters}

Potential clinical characteristics, including sex, age, body mass index (BMI) at recurrence, recurrent stage, interval of recurrence, concurrent chemotherapy during reirradiation, necrosis before re-irradiation, initial total radiation dose and fractionated dose, re-irradiation prescription dose to the GTV, mean re-irradiation dose, reirradiation fractionated dose, duration of re-irradiation, accumulated total prescription dose to the GTV (initial and recurrent), and recurrent tumor volume, were recorded. The recurrent stage was reclassified according to the 2009 American Joint Committee on Cancer 
staging system. The interval of recurrence was defined from the last day of initial radiotherapy to the first day of re-irradiation. Necrosis before re-irradiation was defined as necrosis detected before the first day of re-irradiation. Duration of re-irradiation was calculated from the first day to the last day of re-irradiation.

\section{Statistical methods}

All statistical analyses were performed using SPSS 22.0 software (SPSS, Chicago, IL, USA). Receiver operating characteristic curves based on LNN were applied to determine the cutoff points for the continuous variables. Logistic regression analysis was performed to estimate odds ratios (ORs) or adjusted odds ratios (AORs), 95\% Confidence Intervals (CIs) and $P$ values. Univariate logistic regression analysis was performed to identify potential risk factors for LNN. Multivariate logistic regression analysis was performed to distinguish the independent risk factors for LNN from the variables with statistical significance in the univariate logistic regression analysis. Multivariate logistic regression analysis was also used to construct a predictive model for LNN. All statistical tests were two-sided. $P$ values less than 0.05 were considered statistically significant.

\section{Results}

\section{Clinical characteristics}

In total, 228 patients were included in this study; among these, 24 patients were excluded from further analyses because the initial radiation dose was not available. Of the eligible 204 patients, 169 (82.8\%) were diagnosed based on pathologic analysis with locally recurrent NPC; the remaining 35 (17.2\%) were clinically diagnosed based on symptoms, MRI, or CT images. The median BMI of patients was $21.8 \mathrm{~kg} / \mathrm{m}^{2}$ (range, $14.2-31.3 \mathrm{~kg} / \mathrm{m}^{2}$ ), with a median age of 46 years (range, $21-79$ years). All patients underwent conventional radiotherapy with 2 Gy/fraction per day, 5 days every week, in the initial treatment; the median isocenter dose to the nasopharynx was $70 \mathrm{~Gy}$ (range, 56-83 Gy). The median interval of recurrence was 30 months (range, 9-216 months). All patients completed the full-course radical IMRT of re-irradiation with the median re-irradiation prescription dose to the GTV of 64 Gy (range, 58-70 Gy), 2.14 Gy (range, 1.88-2.33 Gy)/ fraction per day, 5 days every week. The duration of reirradiation was 19-61 days (median, 43 days). The mean re-irradiation GTV dose was 61.63-77.44 Gy (median, $69.23 \mathrm{~Gy}$ ), with a median recurrent volume of $43.81 \mathrm{~cm}^{3}$ (range, 2.98-197.99 $\mathrm{cm}^{3}$ ). During follow-up, 77 patients (37.7\%) were diagnosed with nasopharyngeal necrosis; of these, 51 had died. Of those patients who had died, 31 $(60.8 \%)$ died of intractable epistaxis, which was determined to be LNN; 6 (11.8\%) died of distant metastasis;
11 (21.5\%) died of local-regional recurrence; and 3 (5.9\%) died of sequelae, such as difficulty in feeding, encephalatrophy, and other medical complications. Cutoff points of the continuous variables were analyzed based on LNN; the distribution of patients according to the cutoff points is shown in Table 1. The respective manifestations of nasopharyngeal necrosis on MRI, pathologic examination, and endoscopy are shown in Figs. 1, 2 and 3.

\section{Univariate logistic regression analysis}

Clinical characteristics, including sex, age, BMI at recurrence, recurrent stage, interval of recurrence, concurrent chemotherapy during re-irradiation, necrosis before re-irradiation, initial total radiation dose and fractionated dose, re-irradiation prescription dose to the GTV, mean re-irradiation dose, re-irradiation fractionated dose, duration of re-irradiation, accumulated total prescription dose to the GTV, and recurrent tumor volume, were subjected to univariate logistic regression analysis to determine their associations with LNN. Binary univariate regression analysis showed that female sex (OR 3.308; 95\% CI 1.468-7.454; $P=0.004$ ), necrosis before re-irradiation (OR 3.136; 95\% CI 1.374-7.160; $P=0.007$ ), mean re-irradiation dose $\geq 71.97$ Gy (OR 2.481; 95\% CI 1.118-5.509; $P=0.026)$, accumulated total GTV dose $\geq 145.50$ Gy (OR 3.220; 95\% CI 1.108-9.357; $P=0.032$ ), and recurrent tumor volume $\geq 25.38 \mathrm{~cm}^{3}$ (OR 4.467; 95\% CI 1.30-15.322; $P=0.017$ ) were associated with LNN (Table 2).

\section{Multivariate regression analysis and the predictive model}

To determine the independent risk factors for LNN, the five factors that were statistically significant were included in the multivariate regression analysis model with Enter method. Sex (AOR 3.354; 95\% CI 1.367-8.231; $P=0.008$ ), necrosis before re-irradiation (AOR 3.469; 95\% CI 1.382-8.710; $P=0.008)$, accumulated total GTV dose (AOR 3.763; 95\% CI 1.041-13.611; $P=0.043$ ), and recurrent tumor volume (AOR 6.063; 95\% CI 1.56123.543; $P=0.009$ ) were the independent risk factors for LNN (Table 2). The corresponding regression equation was as below:

Logit $\pi=1.229 \mathrm{X}_{1}+1.276 \mathrm{X}_{2}+1.548 \mathrm{X}_{3}+1.827 \mathrm{X}_{4}-4.072$

Note: $X_{1}=\operatorname{sex}($ men $=0$; women $=1) ; X_{2}=$ necrosis before re-irradiation (no $=0$; yes $=1$ ); $\mathrm{X}_{3}=$ total GTV dose $(<141.50 \mathrm{~Gy}=0 ; \geq 141.50 \mathrm{~Gy}=1)$; and $\mathrm{X}_{4}=$ recurrent volume $\left(<25.38 \mathrm{~cm}^{3}=0 ; \geq 25.38 \mathrm{~cm}^{3}=1\right)$. Logit $\pi=\ln (\pi / 1-\pi) ; \pi$ means the risk of developing LNN of corresponding logit value.

The logit $\pi$ value for each patient was calculated according to the scoring model and graded quarterly by 
Table 1 Characteristics of 204 patients with locally recurrent nasopharyngeal carcinoma

\begin{tabular}{|c|c|c|c|}
\hline Characteristic & All $(n=204)$ & $\operatorname{LNN}(n=31)$ & Non-LNN $(n=173)$ \\
\hline \multicolumn{4}{|l|}{ Sex } \\
\hline Men & $160(78.4)$ & $18(58.1)$ & $142(82.1)$ \\
\hline Women & $44(21.6)$ & $13(41.9)$ & $31(17.9)$ \\
\hline \multicolumn{4}{|l|}{ Age (years) } \\
\hline$<39.5$ & $48(23.5)$ & $4(12.9)$ & $44(25.4)$ \\
\hline$\geq 39.5$ & $156(76.5)$ & $27(87.1)$ & $129(74.6)$ \\
\hline \multicolumn{4}{|c|}{ Initial radiation dose (Gy) } \\
\hline$<74.68$ & $178(87.3)$ & $25(80.6)$ & $153(88.4)$ \\
\hline$\geq 74.68$ & $26(12.7)$ & $6(19.4)$ & $20(11.6)$ \\
\hline \multicolumn{4}{|l|}{ Recurrent stage } \\
\hline । & $13(6.4)$ & $0(0)$ & $13(7.5)$ \\
\hline$\|$ & $25(12.3)$ & $4(12.9)$ & $21(12.1)$ \\
\hline III & $78(38.2)$ & $13(41.9)$ & $65(37.6)$ \\
\hline IV & $88(43.1)$ & $14(45.2)$ & $74(42.8)$ \\
\hline \multicolumn{4}{|c|}{ Interval of recurrence (months) } \\
\hline$<25.5$ & $73(35.8)$ & $9(29.0)$ & $64(37.0)$ \\
\hline$\geq 25.5$ & $131(64.2)$ & $22(72.0)$ & $109(63.0)$ \\
\hline \multicolumn{4}{|c|}{ CCRT during re-irradiation } \\
\hline Yes & $67(32.8)$ & $8(25.8)$ & $59(34.1)$ \\
\hline No & $137(67.2)$ & $23(74.2)$ & $114(65.9)$ \\
\hline \multicolumn{4}{|c|}{ Necrosis before re-irradiation } \\
\hline Yes & $41(20.1)$ & $12(38.7)$ & $29(16.8)$ \\
\hline No & $163(79.9)$ & $19(61.3)$ & $144(83.2)$ \\
\hline \multicolumn{4}{|c|}{ Re-irradiation dose (Gy) } \\
\hline$<67$ & $135(66.2)$ & $18(58.1)$ & $117(67.6)$ \\
\hline$\geq 67$ & $69(33.8)$ & $13(41.9)$ & $56(32.4)$ \\
\hline \multicolumn{4}{|c|}{ Mean re-irradiation dose (Gy) } \\
\hline$<71.97$ & $152(74.5)$ & $18(58.1)$ & $134(77.5)$ \\
\hline$\geq 71.97$ & $52(25.5)$ & $13(41.9)$ & $39(22.5)$ \\
\hline \multicolumn{4}{|c|}{ Re-irradiation fractionated dose (Gy) } \\
\hline$<2.3$ & $197(96.6)$ & $30(96.8)$ & $167(96.5)$ \\
\hline$\geq 2.3$ & $7(3.4)$ & $1(3.2)$ & $6(3.5)$ \\
\hline \multicolumn{4}{|c|}{ Duration of re-irradiation (days) } \\
\hline$<49.5$ & $173(84.8)$ & $23(74.2)$ & $150(86.7)$ \\
\hline$\geq 49.5$ & $31(15.2)$ & $8(25.8)$ & $23(13.3)$ \\
\hline \multicolumn{4}{|c|}{ Accumulated total GTV dose (Gy) } \\
\hline$<141.5$ & $186(91.2)$ & $25(80.6)$ & $161(93.1)$ \\
\hline$\geq 141.5$ & $18(8.8)$ & $6(19.4)$ & $12(6.9)$ \\
\hline \multicolumn{4}{|c|}{ Recurrent tumor volume $\left(\mathrm{cm}^{3}\right)$} \\
\hline$<25.38$ & $59(28.9)$ & $3(9.7)$ & $56(32.4)$ \\
\hline$\geq 25.38$ & $145(71.1)$ & $28(90.3)$ & $117(67.6)$ \\
\hline \multicolumn{4}{|l|}{ BMI } \\
\hline$<19.8$ & $50(24.5)$ & $6(19.4)$ & $44(25.4)$ \\
\hline$\geq 19.8$ & $154(75.5)$ & $25(80.6)$ & $129(74.6)$ \\
\hline \multicolumn{4}{|l|}{ Necrosis } \\
\hline Yes & $77(37.7)$ & $31(100)$ & $46(26.6)$ \\
\hline No & $127(62.2)$ & $0(0)$ & $127(73.4)$ \\
\hline
\end{tabular}

All values are presented as the number of patients followed by percentage in the parentheses

LNN lethal nasopharyngeal necrosis, CCRT concurrent chemoradiotherapy, GTV gross tumor volume, $B M I$ body mass index the corresponding estimated risk of developing LNN (Table 3 ). The probability of patients with logit $\geq 1.099$ developing LNN was over $75 \%$ after re-irradiation with radical IMRT. However, the probability of developing LNN was less than $25 \%$ when logit $\pi<-1.099$. In this study, 12 patients were assigned to the high-risk group which was defined as risk degree IV, and seven patients actually developed LNN (Table 3).

\section{Discussion}

In the present study, for patients with NPC who were treated with radical IMRT, we found that sex, necrosis before re-irradiation, accumulated total prescription dose to the GTV, and recurrent tumor volume are risk factors for LNN. We therefore constructed a feasible prognostic model that included these factors to predict the risk of developing LNN.

IMRT has been proven to improve tumor control and decrease acute and late toxicities when compared with conventional radiation technology [7, 8, 10, 20-22]. However, some severe late toxicities of IMRT, especially LNN, have adversely affected patients' quality of life and survival. According to previous studies, $11 \%-32 \%$ of NPC patients with severe late adverse events underwent re-irradiation with IMRT, which resulted in nasopharyngeal necrosis $[9,15,21]$. Even worse, approximately $45 \%$ of nasopharyngeal necrosis later involved the internal carotid artery and resulted in LNN [22]. Hemostasis by gelatin sponge compression and nasopharyngeal packing through the anterior and/or posterior nares are the standard therapies. Internal/external carotid artery ligation, stent implantation, and other surgery treatments are applied when necessary [23-27]. Unfortunately, the cure rate remains very low despite the hemostasis. It is urgent to identify re-irradiated NPC patients who are at high risk of developing LNN.

Many studies cited dosage as an important risk factor for the severity of necrosis $[13-15,17,19,23]$. Bedwinek et al. [19] reported that osteonecrosis occurred in $9 \%$ of patients with oral carcinoma and NPC who received radiotherapy if the dose was more than $70 \mathrm{~Gy}$. Similarly, Mark et al. [17] observed that $22 \%$ of patients who received a dose greater than 75 Gy experienced osteoradionecrosis. In their study, 28 patients (18.4\%) experienced nasopharyngeal necrosis after initial irradiation with a dose over $70 \mathrm{~Gy}$. However, the incidence was much higher for re-irradiated patients. Hua et al. [15] reported that 14 of $28(50 \%)$ patients developed nasopharyngeal necrosis after re-irradiation with an accumulated prescription dose over $120 \mathrm{~Gy}$. In our study, univariate analysis showed that both the accumulated prescription dose to the GTV and the mean re-irradiation dose were statistically related to LNN, and multivariate analysis showed 


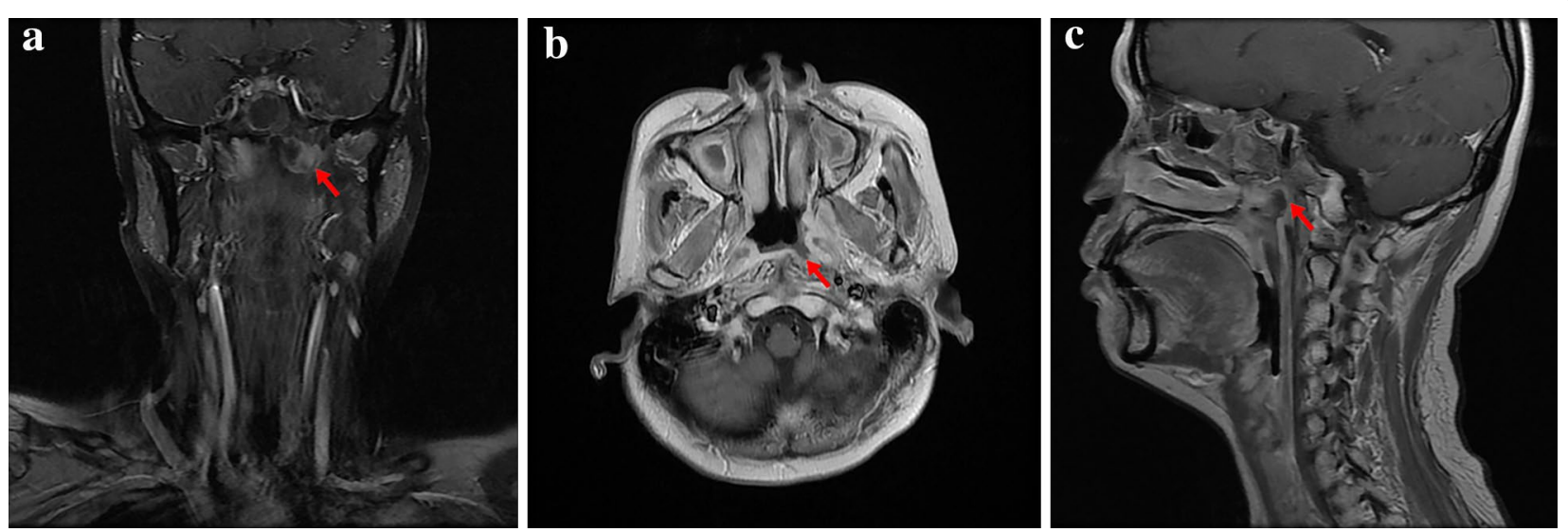

Fig. 1 Magnetic resonance imaging of nasopharyngeal necrosis. a Coronal, contrast-enhanced, T1-weighted image shows non-enhanced soft tissues (like a hole) mixed with tiny air bubbles in the left nasopharyngeal lateral recess (arrow). b Transverse, contrast-enhanced, T1-weighted image shows the necrotic extent in the nasopharyngeal posterior wall (arrow). c Sagittal, contrast-enhanced, T1-weighted image shows an obvious defect in the left nasopharyngeal lateral recess (arrow)
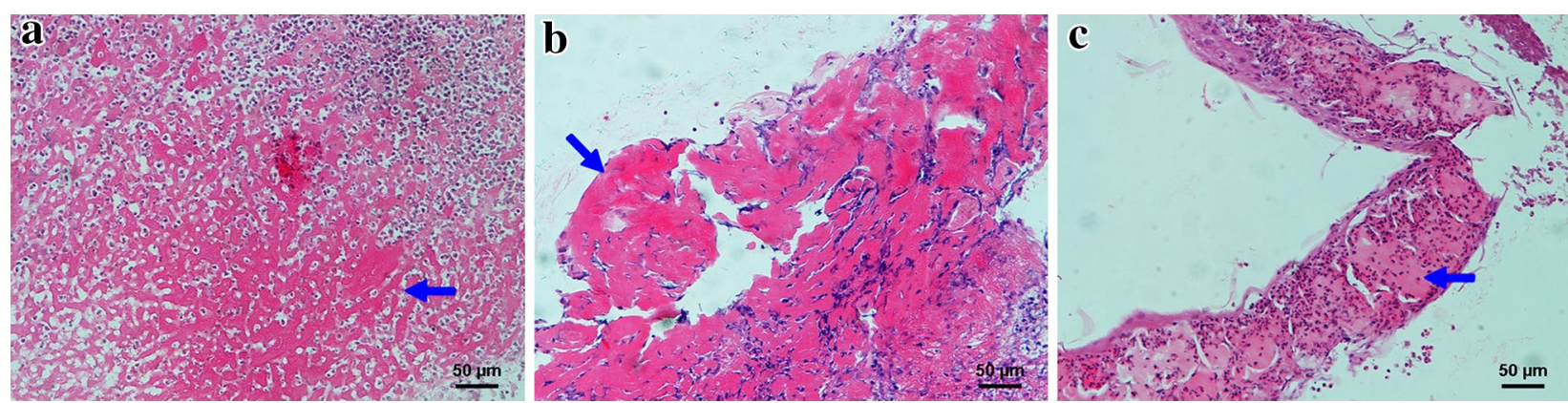

Fig. 2 Pathologic characteristics of nasopharyngeal necrosis. The three necrotic tissues (a-c) were taken from three NPC patients. Hematoxylineosin staining of nasopharyngeal necrosis is similar that shows many red-stained substances without cellular structure (arrows)

that the accumulated prescription dose to the GTV was an independent risk factor for LNN. Additionally, large recurrent tumor volume is usually considered one of the independent factors of poor survival in recurrent NPC [28]. Han et al. [10] showed that the OS of NPC patients with small recurrent tumor volume $\left(\leq 38 \mathrm{~cm}^{3}\right)$ was 1.6 times longer than that of patients with larger recurrent tumor volume $\left(>38 \mathrm{~cm}^{3}\right)$ when treated with IMRT. Moreover, Hua et al. [11] found that the recurrent tumor volume $>42 \mathrm{~cm}^{3}$ was an independent predictor of OS in patients with locally recurrent NPC who were re-irradiated with IMRT. In our study, we found that a recurrent tumor volume $\geq 25.38 \mathrm{~cm}^{3}$ was an independent prognostic factor for LNN; this tumor volume was much lower than those in previous reports discussing survival status, suggesting that re-irradiation of large recurrent tumors should be administered with caution. Re-irradiation is usually the last chance for cure after a patient's first relapse; therefore, for patients at high risk of developing
LNN, decreasing the re-irradiation dose or the recurrent tumor volume might be feasible methods to prevent LNN. Considering that reducing the re-irradiation dose would likely result in worse control of NPC recurrence, administering neoadjuvant chemotherapy to decrease the tumor volume may lower the risk of developing LNN and improve the local control rate and the survival rate [29, 30]. Future clinical trials combining chemotherapy, molecular targeted therapy, and radiotherapy are expected to determine the optimal re-irradiation dose for patients with recurrent NPC.

A generally accepted mechanism of injury after irradiation is a hypovascular-hypoxic-hypocellular condition that causes the breakdown of local tissue, exposing bone, and the formation of sequestra [31]. The inflammation caused by this non-healing wound may, in turn, increase the demand of the local tissue for energy, oxygen, and other metabolites, which may lead to more serious collagen destruction and cell death [17]. This is a 

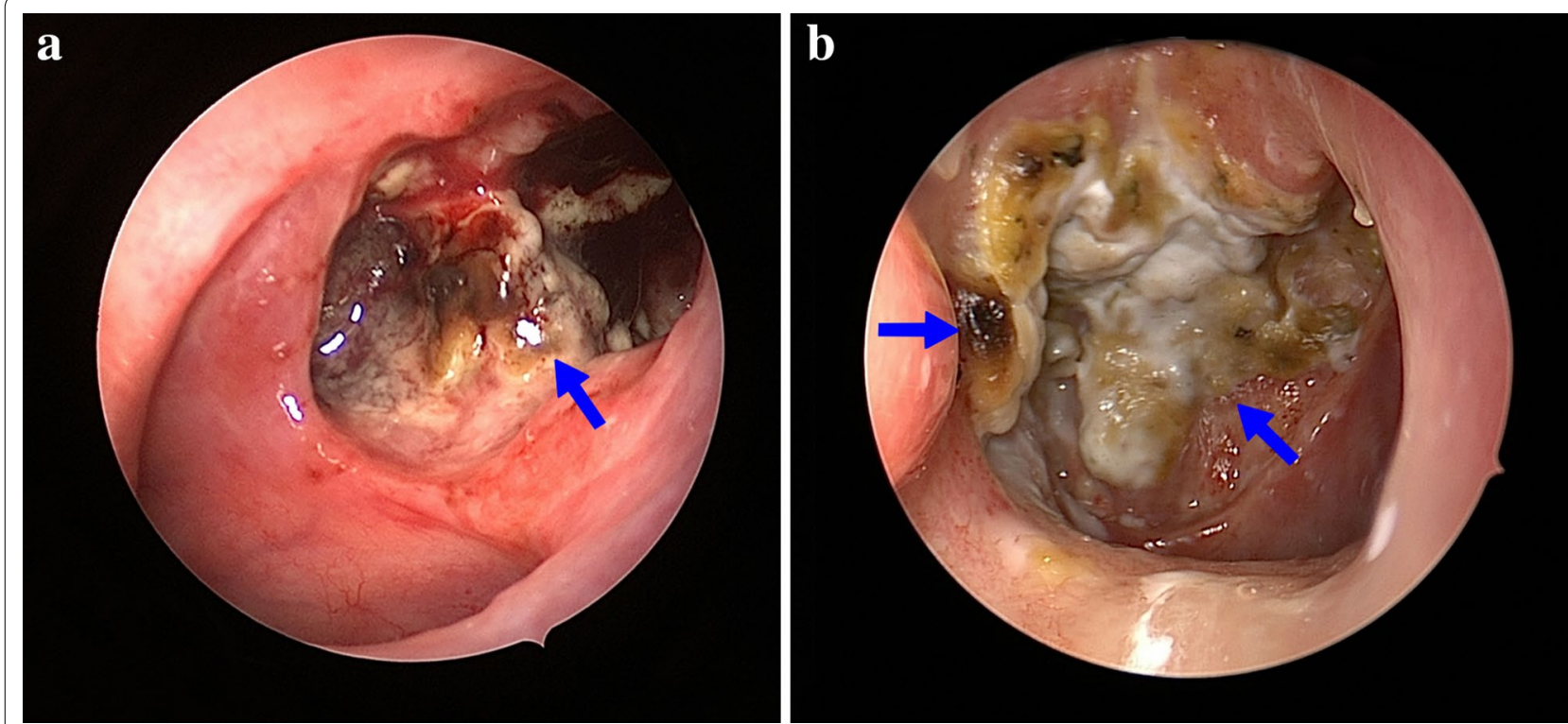

Fig. 3 Endoscopic examination of nasopharyngeal necrosis. Nasopharyngeal necrosis is shown under endoscopic examination (arrows). a The necrosis is located in the roof, posterior, and left wall with obvious mucosa ulcer. Sequestra and necrotic bones can also be seen in the nasopharyngeal cavity. b The nasopharyngeal cavity is covered with yellow to black purulent secretion with necrosis located in the roof, posterior, and right lateral wall of the nasopharynx

Table 2 Univariate and multivariate regression analysis on the association between patient characteristics and LNN

\begin{tabular}{|c|c|c|c|c|c|c|}
\hline \multirow[t]{2}{*}{ Variable } & \multicolumn{3}{|c|}{ Univariate analysis } & \multicolumn{3}{|c|}{ Multivariate analysis } \\
\hline & OR & $95 \% \mathrm{Cl}$ & $P$ & AOR & $95 \% \mathrm{Cl}$ & $P$ \\
\hline Sex (women vs. men) & 3.308 & $1.468-7.454$ & 0.004 & 3.354 & $1.367-8.231$ & 0.008 \\
\hline Age ( $\geq 39.5$ vs. $<39.5$ years) & 2.302 & $0.763-6.947$ & 0.139 & - & - & - \\
\hline Initial radiation dose ( $\geq 74.68$ vs. $<74.68$ Gy) & 1.836 & $0.672-5.018$ & 0.236 & - & - & - \\
\hline Recurrent stage (I vs. || vs. ||| vs. IV) & - & - & 0.999 & - & - & - \\
\hline Interval of recurrence ( $\geq 25.5$ vs. $<25.5$ months) & 1.435 & $0.623-3.207$ & 0.396 & - & - & - \\
\hline СCT during re-irradiation (yes vs. no) & 0.672 & $0.283-1.594$ & 0.367 & - & - & - \\
\hline Necrosis before re-irradiation (yes vs. no) & 3.136 & $1.374-7.160$ & 0.007 & 3.469 & $1.382-8.710$ & 0.008 \\
\hline Re-irradiation GTV dose ( $\geq 67$ vs. < 67 Gy) & 1.509 & $0.691-3.296$ & 0.302 & - & - & - \\
\hline Mean re-irradiation dose ( $\geq 71.97$ vs. < 71.97 Gy) & 2.481 & $1.118-5.509$ & 0.026 & 1.713 & $0.691-4.248$ & 0.246 \\
\hline Re-irradiation fractionated dose ( $\geq 2.3$ vs. $<2.3$ Gy) & - & - & 0.999 & - & - & - \\
\hline Duration of re-irradiation ( $\geq 49.5$ vs. $<49.5$ days) & 2.268 & $0.907-5.672$ & 0.08 & - & - & - \\
\hline Accumulated total GTV dose ( $\geq 141.5$ vs. $<141.5$ Gy) & 3.220 & $1.108-9.357$ & 0.032 & 3.763 & $1.041-13.611$ & 0.043 \\
\hline Recurrent tumor volume ( $\geq 25.38$ vs. $<25.38 \mathrm{~cm}^{3}$ ) & 4.467 & $1.302-15.322$ & 0.017 & 6.063 & $1.561-23.543$ & 0.009 \\
\hline BMI ( $\geq 19.8$ vs. $<19.8)$ & 1.421 & $0.547-3.691$ & 0.470 & - & - & - \\
\hline
\end{tabular}

$L N N$ lethal nasopharyngeal necrosis, $C l$, confidence interval, $C C T$ concurrent chemoradiotherapy, GTV gross tumor volume, $B M I$ body mass index, $O R$ odds ratio, $A O R$ adjusted odds ratio

reasonable explanation for our findings. We found that necrosis before re-irradiation was an independent risk factor for LNN. Of the 41 patients with nasopharyngeal necrosis after the first irradiation, 12 (29.3\%) progressed to LNN after re-irradiation with IMRT. Re-irradiation aggravated injuries caused by the first irradiation, which increased oxygen demand in these aggravated areas. At the same time, previous repair processes, such as fibrosis, may also constrain the local blood supply, which can hinder the healing of necrosis after re-irradiation. As previously reported, weekly debridement and excision of necrotic tissue under nasopharyngeal endoscopy, daily 
Table 3 The risk of developing LNN according to the model

\begin{tabular}{llllc}
\hline Risk degree & No. of total patients & Risk of developing LNN & Value of logitm & No. of LNN patients \\
\hline I & 56 & $<25 \%$ & $<-1.099$ & 1 \\
II & 90 & $25 \%-50 \%$ & -1.099 to 0 & 11 \\
III & 46 & $50 \%-75 \%$ & $0-1.099$ & 12 \\
IV & 12 & $\geq 75 \%$ & $\geq 1.099$ & 7
\end{tabular}

Logitr was calculated from the model and classified into four degrees according to the corresponding risk of developing LNN

LNN lethal nasopharyngeal necrosis

nasopharyngeal irrigation, intravenous nutrition, and systematic antibiotic therapy can improve some cases to a certain extent [15], which may diminish the need for procedures to treat nasopharyngeal necrosis. Thus, once nasopharyngeal necrosis is diagnosed, timely and effective treatment is necessary.

Finally, we found that women were more likely than men to develop LNN, suggesting that intrinsic biological traits, such as sensitivity to radiation, repair ability, and hormone levels, may contribute to a patient's likelihood of developing LNN. Future investigations should study the relationship between necrosis and sex.

This study had several limitations. First, quick and effective measures are very important for hemorrhage rescue, but treatments of massive nasopharyngeal bleeding varied considering expense, distance, and clinical medical condition. Massive nasopharyngeal bleeding involving the internal maxillary artery could be rescued with effective treatment, such as hemostasis by gelatin sponge compression, nasopharyngeal packing, artery ligation, and other surgical measures [32]. However, carotid artery rupture is a common result of LNN, which results in a high mortality [15]. In this study, we could not analyze ruptured arteries because of insufficient clinical data. Second, this study had a relatively small patient population, which diminishes the relevance of the results. Last, as a retrospective study, the patient population's clinical characteristics were diverse; therefore, an observational prospective study is necessary to validate this scoring system.

\section{Conclusions}

For patients with NPC who underwent radical re-irradiation with IMRT, we devised a model, which includes sex, necrosis before re-irradiation, accumulated total prescription dose to the GTV, and recurrent tumor volume, which can effectively predict the risk of developing LNN. We expect that future investigations will suggest strategies to prevent or reverse the development of LNN.

\section{Authors' contributions}

YQX and XG contributed to conception and design of the research and revised the manuscript, YHY, WXX, and JLS drafted the manuscript and analysis data;
WJM, YL, and YFY contributed to analysis and interpretation of data. HL, LRK, and JY participated in data acquisition and literature research and revised the manuscript. All authors read and approved the final manuscript.

\section{Author details}

${ }^{1}$ State Key Laboratory of Oncology in South China, Collaborative Innovation Center of Cancer Medicine, Sun Yat-sen University Cancer Center, Guangzhou 510060, Guangdong, P. R. China. ${ }^{2}$ Department of Nasopharyngeal Carcinoma, Sun Yat-sen University Cancer Center, Guangzhou 510060, Guangdong, P. R. China. ${ }^{3}$ Department of Biodevices and Diagnostics, Institute of Bioengineering and Nanotechnology, Singapore 138669, Singapore. ${ }^{4}$ Department of Radiation Therapy, 3rd Affiliated Hospital of Guangzhou Medical University, Guangzhou 510150, Guangdong, P. R. China. ${ }^{5}$ Department of Pathology, Sun Yat-sen University Cancer Center, Guangzhou 510060, Guangdong, P. R. China. ${ }^{6}$ Department of Biostatistics and Epidemiology, School of Public Health, Sun Yat-sen University, Guangzhou 510080, Guangdong, P. R. China.

\section{Acknowledgements}

This work was supported by the National Natural Science Foundation of China (No. 81472525 and 81572665) and the Science and Technology Planning

Project of Guangdong Province, China (No. 2014A050503033).

\section{Competing interests}

The authors declare that they have no competing interests.

Received: 2 November 2015 Accepted: 14 June 2016

Published online: 29 June 2016

References

1. Wei KR, Zheng RS, Zhang SW, Liang ZH, Ou ZX, Chen WQ. Nasopharyngeal carcinoma incidence and mortality in China in 2010. Chin J Cancer 2014;33(8):381-7.

2. Cao SM, Xu YJ, Lin GZ, Huang QH, Wei KR, Xie SH, et al. Estimation of cancer burden in Guangdong Province, China in 2009. Chin J Cancer. 2015;34(3):58

3. Wei WI, Sham JS. Nasopharyngeal carcinoma. Lancet. 2005:365(9476):2041-54

4. Lee AW, Poon YF, Foo W, Law SC, Cheung FK, Chan DK, et al. Retrospective analysis of 5037 patients with nasopharyngeal carcinoma treated during 1976-1985: overall survival and patterns of failure. Int J Radiat Oncol Biol Phys. 1992;23(2):261-70.

5. Wee J, Tan EH, Tai BC, Wong HB, Leong SS, Tan T, et al. Randomized trial of radiotherapy versus concurrent chemoradiotherapy followed by adjuvant chemotherapy in patients with American Joint Committee on Cancer/ International Union against cancer stage III and IV nasopharyngeal cancer of the endemic variety. J Clin Oncol. 2005;23(27):6730-8.

6. Suarez C, Rodrigo JP, Rinaldo A, Langendijk JA, Shaha AR, Ferlito A. Current treatment options for recurrent nasopharyngeal cancer. Eur Arch Otorhinolaryngol. 2010;267(12):1811-24.

7. Lu TX, Mai WY, Teh BS, Zhao C, Han F, Huang Y, et al. Initial experience using intensity-modulated radiotherapy for recurrent nasopharyngeal carcinoma. Int J Radiat Oncol Biol Phys. 2004:58(3):682-7.

8. Hsiung CY, Yorke ED, Chui CS, Hunt MA, Ling CC, Huang EY, et al. Intensitymodulated radiotherapy versus conventional three-dimensional 
conformal radiotherapy for boost or salvage treatment of nasopharyngeal carcinoma. Int J Radiat Oncol Biol Phys. 2002;53(3):638-47.

9. Chen HY, Ma XM, Ye M, Hou YL, Xie HY, Bai YR. Effectiveness and toxicities of intensity-modulated radiotherapy for patients with locally recurrent nasopharyngeal carcinoma. PLOS ONE. 2013;8(9):e73918.

10. Han F, Zhao C, Huang SM, Lu LX, Huang Y, Deng XW, et al. Long-term outcomes and prognostic factors of re-irradiation for locally recurrent nasopharyngeal carcinoma using intensity-modulated radiotherapy. Clin Oncol. 2012;24(8):569-76.

11. Hua YJ, Han F, Lu LX, Mai HQ, Guo X, Hong MH, et al. Long-term treatment outcome of recurrent nasopharyngeal carcinoma treated with salvage intensity modulated radiotherapy. Eur J Cancer. 2012;48(18):3422-8.

12. Prescribing, recording, and reporting proton-beam therapy (supplement to ICRU Report 50), ICRU Report 62. ICRU, Bethesda; 1999. http://www. icru.org.

13. Chen MY, Mai HQ, Sun R, Guo X, Zhao C, Hong MH, et al. Clinical findings and imaging features of 67 nasopharyngeal carcinoma patients with postradiation nasopharyngeal necrosis. Chin J Cancer. 2013;32(10):533-8.

14. Huang $X M$, Zheng YQ, Zhang XM, Mai HQ, Zeng L, Liu X, et al. Diagnosis and management of skull base osteoradionecrosis after radiotherapy for nasopharyngeal carcinoma. Laryngoscope. 2006;1 16(9):1626-31.

15. Hua YJ, Chen MY, Qian CN, Hong MH, Zhao C, Guo L, et al. Postradiation nasopharyngeal necrosis in the patients with nasopharyngeal carcinoma. Head Neck. 2009;31(6):807-12.

16. Chin SC, Jen YM, Chen CY, Som PM. Necrotic nasopharyngeal mucosa: an ominous MR sign of a carotid artery pseudoaneurysm. AJNR Am J Neuroradiol. 2005;26(2):414-6.

17. Marx RE. A new concept in the treatment of osteoradionecrosis. J Oral Maxillofac Surg. 1983;41(6):351-7.

18. Lee AW, Law SC, Ng SH, Chan DK, Poon YF, Foo W, et al. Retrospective analysis of nasopharyngeal carcinoma treated during 1976-1985: late complications following megavoltage irradiation. Br J Radiol. 1992:65(778):918-28.

19. Bedwinek JM, Shukovsky LJ, Fletcher GH, Daley TE. Osteonecrosis in patients treated with definitive radiotherapy for squamous cell carcinomas of the oral cavity and naso-and oropharynx. Radiology. 1976;119(3):665-7.

20. Chua DT, Sham JS, Leung LH, Au GK. Re-irradiation of nasopharyngeal carcinoma with intensity-modulated radiotherapy. Radiother Oncol. 2005:77(3):290-4
21. Qiu S, Lin S, Tham IW, Pan J, Lu J, Lu JJ. Intensity-modulated radiation therapy in the salvage of locally recurrent nasopharyngeal carcinoma. Int J Radiat Oncol Biol Phys. 2012;83(2):676-83.

22. Tian YM, Guan Y, Xiao WW, Zeng L, Liu S, Lu TX, et al. Long-term survival and late complications in intensity-modulated radiotherapy of locally recurrent T1-T2 nasopharyngeal carcinoma. Head Neck. 2016;38(2):225-31.

23. Lam JW, Chan JY, Lui WM, Ho WK, Lee R, Tsang RK. Management of pseudoaneurysms of the internal carotid artery in postirradiated nasopharyngeal carcinoma patients. Laryngoscope. 2014;124(10):2292-6.

24. Auyeung KM, Lui WM, Chow LC, Chan FL. Massive epistaxis related to petrous carotid artery pseudoaneurysm after radiation therapy: emergency treatment with covered stent in two cases. AJNR Am J Neuroradiol. 2003;24(7):1449-52

25. Wong GK, Chan KK, Yu SC, Tsang RK, Poon WS. Treatment of profuse epistaxis in patients irradiated for nasopharyngeal carcinoma. ANZ J Surg. 2007;77(4):270-4

26. Ellens DJ, Hurley MC, Surdel D, Shaibani A, Pelzer H, Bendok BR. Radiotherapy-induced common carotid pseudoaneurysm presenting with initially occult upper airway hemorrhage and successfully treated by endovascular stent graft. Am J Otolaryngol. 2010;31(3):195-8.

27. Klotz DA, Winkle MR, Richmon J, Hengerer AS. Surgical management of posterior epistaxis: a changing paradigm. Laryngoscope. 2002;112(9):1577-82.

28. Wu SX, Chua DT, Deng ML, Zhao C, Li FY, Sham JS, et al. Outcome of fractionated stereotactic radiotherapy for 90 patients with locally persistent and recurrent nasopharyngeal carcinoma. Int J Radiat Oncol Biol Phys. 2007:69(3):761-9.

29. Oh JL, Vokes EE, Kies MS, Mittal BB, Witt ME, Weichselbaum RR, et al. Induction chemotherapy followed by concomitant chemoradiotherapy in the treatment of locoregionally advanced nasopharyngeal cancer. Ann Oncol. 2003;14(4):564-9.

30. Rischin D, Corry J, Smith J, Stewart J, Hughes P, Peters L. Excellent disease control and survival in patients with advanced nasopharyngeal cancer treated with chemoradiation. J Clin Oncol. 2002;20(7):1845-52.

31. Fajardo LF. Basic mechanisms and general morphology of radiation injury Semin Roentgenol. 1993;28(4):297-302.

32. He CC, Si YF, Xie YA, Yu L. Management of intractable epistaxis in patients who received radiation therapy for nasopharyngeal carcinoma. Eur Arch Otorhinolaryngol. 2013;270(10):2763-7.

\section{Submit your next manuscript to BioMed Central and we will help you at every step:}

- We accept pre-submission inquiries

- Our selector tool helps you to find the most relevant journal

- We provide round the clock customer support

- Convenient online submission

- Thorough peer review

- Inclusion in PubMed and all major indexing services

- Maximum visibility for your research

Submit your manuscript at www.biomedcentral com/submit
BioMed Central 